break the galvanic current by slight pressure of the thumb; $f f$, sockets into which the cannulæ can be fixed by screws; $g$, receptacle for connecting wires from battery; on the long. screw (b) rides the clamp $(h)$, between the jaws of which the ends of the platinum wires can be fixed by a screw. A gag, artery forceps, ligatures, a strong

FIG. 3. whipcord ligature, scalpel, and a couple of Sims wire guides (Fig: 3) complete the apparatus.

The patient being placed on the table, and brought under the influence of the anæsthetic, a vertical submental incision (after Nunneley) is made in the centre of the mylo-hyoid space, and the opening freed into the floor of the mouth in front of the frænum of the tongue. Slight bleeding takes place. The passage is enlarged by means of the two forefingers working against one another sufficiently to allow the nozzle of the cannulæ $(c)$ to pass through. The gag is then placed between the jaws, and the mouth kept open; the whipcord ligature is passed through the raphe about an inch from the tip of the tongue. The cannulæ $(c)$, freed from the handle, and with the wire-loop $(d)$ drawn home, the nozzle of which is then passed through the submental incision up into the mouth. The wire-loop is then, by means of a forceps, drawn out from the nozzle of the cannula to form the loop $(d)$, through which the tongue, with its whipcord ligature, is introduced, and the tongue being gently drawn forwards by an assistant the operator carries the loop $(d)$ back over the root of the tongue close up to the epiglottis. The assistant now gently pulls on the free ends of the platinum-wire, so as to tighten the loop $(d)$, which the operator gets into position by means of his two forefingers, and which he retains in situ by means of the wire-guides (Fig. 3), one on either side of the tongue. Now the cannulæ and the free ends of the platinum-wire are passed through the sockets $(f f)$ of the handle $(a)$; the screws (at $f f$ ) are then screwed tight, and the cannulæ fixed. The ends of the wire are then fixed between the jaws of the clamp $(h)$ by a screw. The screw (b) on which the clamp (h) travels is then gently worked sufficient to fix the loop round the root of the tongue. All being satisfactory, the connecting-wires from the battery are placed and fixed in the receptacles $(g)$, the ivory-nut $(e)$ is now screwed home, the galvanic current is completed, the loop reddens, and the ecraseur is at work. The screw $(b)$ is then gently worked, and, above all, the process of tightening should be very slowly performed. The wire of the écraseur being screwed home only as it becomes loose by cutting through the tissues, any force may break it. The tongue is gradually burnt through in from seven to ten minutes, and, being detached, is drawn out of the mouth through the lips by the assistant. The operator immediately breaks the current by unscrewing the ivory nut $(e)$, and removes the nozzle of the cannulæ from the mylo-hyoid space. If the lingual arteries begin to bleed they are easily seized and tied. The tongue is found severed obliquely from behind forwards. The gag is then released, and the submental incision brought together with strapping; the operation is complete, and the patient taken to bed.

Whenever bleeding follows the operation that has been described, it is from one of two causes : the wire cautery has been used at too great a temperature, or has been screwed up too rapidly. Hæmorrhage is, however, easily restrained, either by the application of iron-lint or ice.

The introduction of the galvanic cautery has so benefited and simplified the operations on the tongue that the surgeon can by its means isolate the disease with ease. He can satisfy himself that the whole tumour is under his control ; his operation is bloodless, and he need in no way expedite his movements. Before the introduction of the galvanic écraseur, operations on the tongue were very rarely performed. Now, however, it is different; they are of common occurrence, and by these means operations on the tongue for cancer, I am inclined to believe, are more suceessful than when they are undertaken by any other means.

Mr. Barwell, in his plan, uses the wire écraseur, and in a complicated manner, to my mind, gets one end of the wire passed into the mouth through a submental incision in the mylo-hyoid space by means of a previously-passed piece of cord, and, encircling the tongue, gets it withdrawn through the hyoid space at the opposite side of the tongue by'means of a previously-passed cord loop, endeavouring to include the lingual-gustatory nerves in the wire. All being secured, it crushes through the tissues of the tongue, severing the sensory nerves "close to the bone." No doubt the wire écraseur, applied as Nunneley of Leeds performed his operation, comes next to the galvanic cautery, but the galvanic cautery has this great advantage over all others--that it gently burns through the tissues, without any dragging or tearing of the neighbouring structures.

\section{ANTISEPTIC TRANSFUSION OF HUMAN BLOOD IN A PATIENT THE SUBJECT OF SECONDARY HAMORRHAGE; CURE.}

BY WILLIAM MACEWEN, M.D., SURGEON AND LECTURER ON CLINICAL SURGERY, ROYAL INFIRMARY,
GLASGOW.

THE following case of transfusion of human blood is worthy of record, on account of the antiseptic precautions which were adopted and the complete success of the operation.

The patient was a man, twenty-three years of age, on whom lithotomy was performed for the removal of a large. spiked oxalate-of-lime calculus. There was little bleeding as an immediate sequent of the operation, and it was completely arrested before he left the table. Half an hour after having been put to bed, the house-surgeon found him to be very comfortable and in a good general state; there was then only slight staining on the sheet under the pelvis. Two hours and a half after he was found to be in a state of complete depletion, from a profuse secondary hæmorrhage which had ensued. The wound was at once plugged. Notwithstanding every attempt to resuscitate him by stimulating drinks, enemata, \&c., it was evident, at the end of three-quarters of an hour, that ground was being fast lost, and it was clear to all present that, if his life was to be saved, something more radical was necessary. Transfusion of blood was proposed. A patient who suffered from injury to the right great toe, and who otherwise was strong and healthy, after being apprised of the slight risk he ran in giving a portion of his blood, freely offered it to his fellow.

The lithotomy patient was then in the following state. He was semi-insensible, could not speak, pulse at the wrist imperceptible, surface of the body blanched and bedewed with a cold perspiration, the lips cream-coloured, and the conjunctival vessels no longer visible. Occasionally he gave a restless feeble toss, accompanied by a deep inspiration.

An attempt to find one of the large veins on the right arm being unsuccessful, the median cephalic of the left was. chosen, and half an inch of its length exposed. An assistant was desired to place the finger of one hand to the distal side of the exposed vein, and to maintain pressure on that part throughout the operation, so as to prevent loss of blood; and with a finger of the other hand, placed on the proximal side about half an inch above the part selected for opening the vessel, he.was to occlude the vein when required. The arm was held well up, so as to empty it of any blood which it might contain. It was also maintained considerably above the level of the patient's body, for three reasons: first, to facilitate the flow of the transfused blood into the trunk; secondly, to prevent the entrance of air into the. body, as the syringe would, with the arm in this position, be necessarily held perpendicularly, with the nozzle downwards, and all contained air would remain at the top of the instrument; and, thirdly, to enable any air to escape which might be in the space intervening between the opening in the vein, and the occluding finger of the assistant on the proximal side. The vein was then opened. Then phle. botomy was performed on the healthy man, the blood being received into a small warm carbolised vessel, from which it was at once drawn into a warm carbolised three-ounce syringe, having a narrow nozzle. When full it was inverted and the piston pressed, so as to expel any air, and the nozzle was then introduced into the vein. A quantity of blood was first injected into the sprce in the vein, between the occluding finger on the proximal side and the opening in the vein itself. When this was done the 
pressure on the proximal side was removed, and the contents of the syringe were slowly injected, until only a couple of drachms remained. The pressure of the assistant's finger was again applied, and the syringe removed. It was then washed in 1 to 80 carbolised watery solution, recharged, and the blood introduced as before. The tin into which the blood flowed was kept free from clot, and several times a fresh cup was substituted. The arm from which the blood was drawn, as well as that into which it was injected, were kept constantly under the spray, and the blood itself, from the time it left the one arm until it was injected into the other, was either exposed to the carbolised spray or in contact with carbolised instruments; so that the whole transfusion was thoroughly antiseptic.

With the exception of the transfusion being performed antiseptically, the other details of the operation were nearly the same as those adopted by Mr. Lister in a case in which he performed transfusion, while I acted as one of the house-surgeons in the Royal Infirmary. That case was reported by me in the Glasgow Medical Journal for Nov. 1869.

Just before the transfusion was begun several of the housesurgeons hinted that the patient had "slipped away." His heart, however, was heard to respond, and the blood was injected. Shortly after the transfusion the gentleman who had his finger over the radial said that, from being imperceptible, it had returned gradually, and had increased until it was distinctly felt. Half an hour after the face had assumed a slight redness, and heat began to be restored to the surface of the body. There were no rigors after the transfusion. Without entering into further detail, it may be said that he slowly but perfectly recovered, and is now a strong, healthy man. He was shown at the Pathological and Clinical Society nine months after the operation, and is still quite well and at work.

Glasgow.

\section{REMARKS ON \\ TWO CASES OF HEBRA'S PRURIGO,} LATELY TREATED AT THE MIDDLESEX HOSPITAL.

By ROBERT LIVEING, M.D., LECTURER ON DISEASES OF THE SKIN AT THE HOSPITAL.

THE prurigo of Hebra, as it is called, has hardly received the attention and recognition that it merits from English observers. Some modern writers are contented to follow Hebra's description without expressing any views or experience of their own on the subject, while others even deny altogether the existence of the malady in this country. There can be little doubt that by many the disease is not recognised from Hebra's description, and indeed this is scarcely to be wondered at. On referring to his account of the disease, we find " in every case the earliest appearance is that of subepidermic papules, as big as hemp-seeds, discovered rather by touch than by sight, since they rise but little above the level of the skin, and do not differ from it at all in colour; they are always isolated, and though they appear in all sorts of places, constantly leave some regions unaffected." Elsewhere he says that these papules are really deep-seated vesicles " formed by a collection of fluid in the deeper layers of the epidermis." Now, English observers have naturally laid considerable stress on this part of Hebra's description; they have constantly looked for, but have generally failed to find these large, papule-like vesicles of the same colour as the surrounding skin, and hence they have often come to the conclusion that the disease does not exist in this country.

There are several reasons why we often fail to find what I may call the original eruption of prurigo. (1) The papules he describes (they have not at all the appearance of vesicles) exist as such only for a very short time; they are quickly injured by the scratching of the patient; this fact, together with the presence of excoriations, eczema, and other changes in the skin, makes it difficult to find them. (2) The large papules, though pale, are not usually of the same colour as the surrounding skin, but of a distinctly redder tint (3) Hebra has, for the purpose of diagnosis, laid too much stress on the elementary form of the eruption, and not enough, by comparison, on the history of the disease, which is, after all, a more important diagnostic feature. With regard to the prurigo papule, I would remark that in elderly people, who have suffered perhaps for many years from chronic eczema of the ordinary kind, we notice that towards the later part of life the eruption often assumes a papular form, and we have, instead of common eczema, a development of large pruriginous papules on the extensor sides of the limbs; these papules are torn and bleeding from the scratching of the sufferer, and present an appearance closely resembling Hebra's prurigo, but with a different history; this modified form of papular eczema is still called prurigo by some writers. But to return. Hebra says that prurigo is not congenital, but commonly appears during infancy. Now it is true that, strictly speaking, it is not congenital; at the same time it always appears at a very early age, generally during the first or second year of life; and this fact I regard as one of the most constant and characteristic features of the disease. I have never met with a single example of its appearance for the first time in an adult, but when once developed it persists through life.

Two cases of this disease have lately been under my care at the Middlesex Hospital, and serve well to illustrate the leading features of the malady. One was in a boy aged about twelve, and the other in a young adult. In the latter case the history and features of the disease were highly characteristic. The eruption had existed from infancy, and had, in spite of all treatment, slowly increased, though varying in severity with the changes of season. It was scattered more or less over the body, but its chief seat was the back of the wrist, arms, thighs, and legs, the latter being very severely affected. The hams, however, were almost entirely free from eruption; so also was the space on the flexor aspect of the elbow-joint. The head and neck were also quite free. The itching, always severe, was described as at times intolerable; the skin of the legs and thighs, which was abnormally dark, was thickened, especially on the outer aspect of the limb, and so hard that it was somewhat difficult to pinch it up between the finger and thumb. On passing the hand quickly over the leg, the harsh, rasp-like character of the skin was very apparent. The general appearance of the eruption on the legs was just such as might be produced by severe persistent phthiriasis leading to chronic, dry, papular eczema, excoriations, pigmentation, and thickening of the skin, but, of course, differing in respect to the parts affected. On a careful examination, it was not difficult to find some of the isolated large pale papules of Hebra, but at this stage of the disease (over twenty years' standing) they are for the most part not isolated, and therefore not recognised. In this case all the leading characters of the disease were present: (1) its development in early infancy, (2) its persistence throughout life, (3) the especial development of the eruption on the lower extremities, with the freedom of the ham, (4) the intolerable itching with the consequent scratching, leading to excoriation, secondary eczema, thickening, and pigmentation.

With regard to treatment, some decided relief was given, of course only temporary, by full doses of arsenic, and soothing local applications.

In conclusion, I would remark that the comparative rarity of this disease in England, even amongst the lower classes, together with what I must call Hebra's exaggerated description of the "large, pale, isolated papule," have led to the erroneous conclusion that the disease does not exist. Lastly, I would point out the fact that in infancy the first appearance of the eruption is usually that recognised as lichen urticatus, which, however, subsequently changes to the eruption characteristic of the disease.

\section{SURGICAL NOTES ON THE ZULU WAR.}

\section{By D. BLAIR BROWN, F.R.C.S. Ed., A.M.D.}

THE following cases and observations, apart from the intense interest shown concerning all appertaining to the Zulu war, will, I think, be found of sufficient interest to find space in THE LANCET.

Assegai Wound of Left Ham.-Private J. H. Mthe Ist Battalion 3rd Regiment Native Contingent, was present on the 12th January at the attack on Sirayo's Kraal. Several prisoners were taken, and were being disarmed. One of them, being irritated by our friendly Kaffirs, 